\title{
Dynamic critical behavior of the one-dimensional $X Y$ model with a long-range interaction
}

\author{
Seong-Gyu Yang, ${ }^{1}$ Hye Jin Park, ${ }^{2}$ and Beom Jun Kim ${ }^{1, *}$ \\ ${ }^{1}$ Department of Physics, Sungkyunkwan University, Suwon 16419, Republic of Korea \\ ${ }^{2}$ Department of Evolutionary Theory, Max Planck Institute for Evolutionary Biology, 24306 Plön, Germany
}

(Received 17 April 2018; revised manuscript received 17 July 2018; published 17 September 2018)

\begin{abstract}
We numerically study dynamic critical behavior of the one-dimensional $X Y$ model with a long-range interaction by using the Monte Carlo method and the resistively-shunted Josephson junction model. The two dynamic models exhibit the mean-field universality class in equilibrium as expected, but the dynamic critical behavior is shown to sensitively depend on details of numerical simulation. In more detail, the trial angle range in the Monte Carlo simulation is found to alter the value of the dynamic critical exponent, and the scaling of the Monte Carlo time unit by the acceptance ratio is shown to be useful to improve the estimation of the dynamic critical exponent. We compare the Monte Carlo result of the dynamic critical exponent with the result from the more realistic dynamic model of the resistively-shunted junction. We conclude that the small value of the trial angle range should be used to properly detect dynamic critical behavior.
\end{abstract}

DOI: 10.1103/PhysRevE.98.032120

\section{INTRODUCTION}

The $X Y$ model is a well-known spin model which has been widely studied in the research field of statistical physics and condensed-matter physics. Unlike the Ising model, the $X Y$ model is described by continuous variables and has a continuous rotational symmetry. According to the MerminWagner theorem [1-3] a system with the continuous symmetry like the $X Y$ model cannot have an ordered phase at any nonzero temperature if the dimensionality of the system is equal or less than two and if the interaction is short ranged. However, in various underlying interaction structures such as a random network [4], a small-world network [5], and a static scale-free network [6], the Mermin-Wagner theorem is not applicable, and the system exhibits a continuous phase transition. The standard values of the static critical exponents in the mean-field (MF) universality class have confirmed in those networks [4-6]. Breaking down of the Mermin-Wagner theorem originates from the underlying long-ranged interaction structure. Since the Mermin-Wagner theorem assumes the local interaction, the system with a long-range interaction does not necessarily satisfy the Mermin-Wagner theorem. Furthermore, if the interaction range becomes comparable to the system size, the universality class of the phase transition becomes MF class, as one can easily understand from the globally coupled system [7]. Hence, the systems undergo the MF phase transition even the system dimension is less than lower critical dimension [7]. It is shown that the $X Y$ model in the one-dimensional lattice with long-range interaction exhibits MF phase transition [7].

Dynamic critical behavior of the $X Y$ model has also been studied in a random and a small-world networks [8,9], with the results consistent with the dynamic MF universality class. The relaxation time scale $\tau$ in a regular $d$-dimensional system

*Corresponding author: beomjun@skku.edu scales with the correlation length $\xi$ as $\tau \sim \xi^{z}$. For a finite system of the linear size $\ell$, the above expression reads $\tau \sim \ell^{z}$ at the critical point. It should be noted that the linear size $\ell$ in networks is ill defined, and thus one often uses instead the total number $N$ of degrees of freedom to get $\tau \sim N^{\bar{z}}$. For a regular lattice with $N=\ell^{d}$, we get the relation $\bar{z}=z / d$ between $z$ and $\bar{z}$. The standard value of the dynamic critical exponent $z=2$ for a relaxational dynamics then corresponds to $\bar{z}=1 / 2$ beyond the upper critical dimension four of the $X Y$ model.

For long-range interaction, however, the dynamic critical behavior has not been well studied in comparison to the static critical behavior. In this paper, we focus on the dynamic critical behavior of the $X Y$ model in the one-dimensional lattice with long-range interaction by using the Monte Carlo (MC) simulations. We also investigate the real-time dynamics of the resistively-shunted junction (RSJ) model which describes the phase variable of the $X Y$ model as the phase of the Cooper pair wave function of the superconducting array $[10,11]$.

The present paper is organized as follows: In Sec. II we introduce the $X Y$ model and describe the RSJ model for dynamics. We investigate the dynamic critical behavior by using the Monte Carlo simulations in Sec. III, by observing dynamic fluctuation in equilibrium (Sec. III A), and by using the so-called the short-time relaxation method (Sec. III B). In Sec. IV, we report numerical results of the RSJ model in comparison to the results in Sec. III. Finally, a conclusion with a brief summary follows in Sec. V.

\section{MODEL}

The $X Y$ model with the interaction range $L$ in onedimensional lattice is described by the Hamiltonian given by

$$
H=-\frac{J}{2 L} \sum_{i=1}^{N} \sum_{j=i-L}^{i+L} \cos \left(\phi_{i}-\phi_{j}\right)
$$


where $J$ is the ferromagnetic interaction strength $(J>0)$, the phase angle $\phi_{i}$ at the $i$ th site is a compact continuous variable in $(-\pi, \pi]$, and the periodic boundary condition is imposed $\left(\phi_{i \pm N}=\phi_{i}\right)$. When $L=1$, we have the typical one-dimensional $X Y$ model with the nearest-neighbor interaction, with no ordered phase at any nonzero temperature. As the interaction range $L$ becomes comparable to $N$, i.e., $L / N \sim \mathcal{O}(1)$, we have the $X Y$ model with a long-range interaction. Hereafter, we use the sufficiently large interaction range $L / N=0.4$ to ensure that the model surely exhibits the MF behavior as shown in Ref. [7].

The upper critical dimension is $d_{u}=4$ for the locally coupled $X Y$ model, and thus the MF results come out when the dimensionality $d$ of the system satisfies $d \geqslant d_{u}$. In this MF regime, the local updating algorithms including the Metropolis MC algorithm is well known to yield the dynamic critical exponent $z=2$ [12], which has been confirmed from numerical studies $[13,14]$. From those existing studies, we also expect to obtain the dynamic critical exponent $\bar{z}=z / d_{u}=$ $1 / 2$ in the one-dimensional $X Y$ model with a long-range interaction. From now on, we call $\bar{z}$ the dynamic critical exponent for convenience.

It is to be emphasized that the equilibrium Hamiltonian (1) alone cannot dictate the temporal dynamic evolution of the system. One can use the Metropolis MC algorithm for the time evolution of the system, and other dynamics can also be used: In Ref. [10], it has been shown that both the timedependent Ginzburg-Landau (TDGL) dynamics and the RSJ dynamics can be used with the same equilibrium Boltzmann distribution for a given Hamiltonian. The RSJ model describes the time evolution of the phase variable $\phi$ of Cooper pair electrons in a superconducting arrays $[10,15,16]$. Although TDGL dynamics describes the time evolution of the same phase variables as in the RSJ dynamics, the two are very different: TDGL dynamics is phenomenologically derived solely from the Hamiltonian and thus TDGL dynamics lacks any justification of the time scale of relaxation dynamics. The other difference is that the thermal noise term in TDGL equation is defined onsite, while the thermal noise currents in RSJ dynamics (see below) are defined as link variables between two superconducting islands. Consequently, the temporal noise correlation takes different forms in TDGL and RSJ dynamics [10]. We note that the RSJ model is based on the current-conservation law applied for the superconducting array and thus can be considered realistic. Although the onedimensional $X Y$ model with a long-range interaction can be very difficult to fabricate in reality by using Josephsoncoupled superconductors, one can still say that RSJ model is more realistic than the Metropolis MC dynamics and the TDGL dynamics.

The sum of all the currents from $i$ th superconductor to neighboring superconductors ( $j$ 's) in the array must be zero, and the current-conservation law can be written as $[10,15,16]$

$$
\sum_{j \in \mathcal{N}(i)}\left[I_{c} \sin \left(\phi_{i}-\phi_{j}\right)+\frac{\hbar}{2 e R} \frac{d}{d t}\left(\phi_{i}-\phi_{j}\right)+\Gamma_{i j}\right]=0
$$

where $\phi_{i}$ is the phase of Cooper pairs at the $i$ th site and $\mathcal{N}(i)$ is the set of neighboring superconductors Josephson coupled to $i$ [11]. The first (the second) term on the left-hand side of Eq. (2) is the supercurrent (the normal resistive current) from $i$ to $j$, where $I_{c}$ and $R$ are the critical current and the normal resistance across a Josephson junction, respectively. We use the conventional notation so that $e(>0)$ and $\hbar$ are the electron charge and the reduced Planck constant, respectively. The third term $\left(\Gamma_{i j}\right)$ is the thermal noise current from $i$ to $j$ which satisfies $\left\langle\Gamma_{i j}(t)\right\rangle=0$ and $\left\langle\Gamma_{i j}(t) \Gamma_{k l}(0)\right\rangle=$ $\left(4 k_{B} T L / R\right)\left(\delta_{i k} \delta_{j l}-\delta_{i l} \delta_{j k}\right) \delta(t)$, where $k_{B}$ is the Boltzmann constant, and $\delta_{i j}$ and $\delta(t)$ are the Kronecker $\delta$ and the Dirac $\delta$, respectively.

We can rewrite Eq. (2) in a dimensionless form [10],

$$
\frac{d \phi_{i}}{d t}=-\sum_{j} G_{i j} \sum_{k \in \mathcal{N}(j)}\left[\frac{1}{2 L} \sin \left(\phi_{j}-\phi_{k}\right)+\eta_{j k}\right],
$$

where we have rescaled time in units of $\hbar /\left(4 e I_{c} R L\right)$ to make $t$ dimensionless, $G_{i j}$ is the lattice Green function for the one-dimensional lattice with the long-range interaction, and $\eta_{i j}$ is the dimensionless thermal noise current defined by $\eta_{i j} \equiv$ $\Gamma_{i j} / 2 L I_{c}$ with conditions $\left\langle\eta_{i j}(t)\right\rangle=0$ and $\left\langle\eta_{i j}(t) \eta_{k l}(0)\right\rangle=$ $2 T\left(\delta_{i k} \delta_{j l}-\delta_{i l} \delta_{j k}\right) \delta(t)$. Note that the temperature $T$ has also been rescaled in units of $\hbar I_{c} / 2 e k_{B}$ to make it dimensionless. The Josephson coupling strength $J$ in Eq. (1) is related with the critical current via $J=\hbar I_{c} / 2 e$, and thus $T$ is now in units of $J / k_{B}$ to make the comparison to the MC results straightforward.

The RSJ equations are the Langevin type and one can map into the Fokker-Planck equation. It is straightforward to get the stationary solution of the Fokker-Planck equation and the equilibrium probability distribution function $P \propto e^{-H / k_{B} T}$ is obtained with the $X Y$ Hamiltonian $H$ in Eq. (1). Accordingly, the phase variable of the $X Y$ model can be directly interpreted as the phase of the superconducting order parameter describing the Cooper pair wave function in superconductivity.

\section{RESULTS: MONTE CARLO DYNAMICS}

In a strict sense, one can observe a thermodynamic phase transition only when the system size is infinite. What we can observe in reality in computational approach is only how the behavior changes as the system size becomes larger. In statistical mechanics, the use of finite sizes to understand what happens in the thermodynamic limit has been well established, and the methodology is often phrased "the finite-size scaling" (FSS). In the present paper, we use two different FSS approaches to investigate the dynamic critical behavior. We first observe the dynamic fluctuation in equilibrium [17-19] and apply the FSS to obtain the dynamic critical exponent. We also use the so-called short-time relaxation method (or the finite-time method) which uses the relaxational behavior in early times $[9,13,14]$. Note that Ref. [20] has suggested the unified framework, called "the finite-size-finite-time scaling," to build a two-dimensional scaling surface rather than a onedimensional scaling curve in the conventional FSS approach.

The $X Y$ model has a continuous $U(1)$ symmetry related with the continuous periodic variable $\phi$. In our MC simulations, we use the the standard Metropolis local updating algorithm. The important parameter in the Metropolis MC simulations of the $X Y$ model is the trial angle range $\Delta \phi$ : The trial angle $\phi_{i}^{\text {try }}$ at the $i$ th site is a uniform random variable 
in the range $\left[\phi_{i}-\Delta \phi, \phi_{i}+\Delta \phi\right]$, and the trial is accepted $\left(\phi_{i}\right.$ is updated to $\left.\phi_{i}^{\text {try }}\right)$ or not $\left(\phi_{i}\right.$ remains unchanged) by using the Metropolis criterion [18]. The trial angle range $\Delta \phi$ affects the acceptance ratio: The smaller $\Delta \phi$, the larger the acceptance ratio. The $\Delta \phi$ dependence of the acceptance ratio can be important in the study of dynamic behavior, since the acceptance ratio alters the time scale of the relaxation. The relaxation can be extremely slow and the acceptance ratio very close to unity if $\Delta \phi$ is very small. Accordingly, dynamic MC results should be carefully analyzed with $\Delta \phi$ dependence of relaxation behavior taken into full account. In our MC study, we examine the dynamics of the system at various values of the trial angle range $\Delta \phi=n(\pi / 6)$ with $n=$ $1,2, \ldots, 6$ for the system sizes $N=50,100,200,400$, and 800. In all measurements in Sec. III A for equilibrium study we perform averages over $10^{5} \mathrm{MC}$ steps with 200 different initial configurations after equilibration for $10^{5} \mathrm{MC}$ steps. In Sec. III B for the short-time relaxation study, averages are made over $5 \times 10^{4}$ independent MC runs.

\section{A. Dynamic fluctuation in equilibrium}

We first present our results for dynamic MC simulations in equilibrium. After a sufficiently long MC simulation for equilibration, measurement in time $t$ is made for a quantity $A(t)$ of interest. We calculate the autocorrelation function $C(t)$ of $A$ defined by $[17,18]$

$$
C(t) \equiv \frac{\left\langle A\left(t+t^{\prime}\right) A\left(t^{\prime}\right)\right\rangle_{t^{\prime}}-\left\langle A\left(t+t^{\prime}\right)\right\rangle_{t^{\prime}}\left\langle A\left(t^{\prime}\right)\right\rangle_{t^{\prime}}}{\left\langle A\left(t^{\prime}\right)^{2}\right\rangle_{t^{\prime}}-\left\langle A\left(t^{\prime}\right)\right\rangle_{t^{\prime}}^{2}},
$$

where $\langle\cdots\rangle_{t^{\prime}}$ denotes the average over the time $t^{\prime}$. At $t=0$, the numerator and the denominator of Eq. (4) are identical to yield $C(t=0)=1$. In other limit of $t \rightarrow \infty, A\left(t+t^{\prime}\right)$ and $A\left(t^{\prime}\right)$ must become independent from each other, and thus we have $C(t \rightarrow \infty)=0$. The relaxation time scale related with the autocorrelation $C(t)$ can be estimated either from the curve fitting to the exponential decay form or from the time integration of $C(t)[17,18]$. We use the latter method and obtain the average relaxation time $\tau$ from

$$
\tau=\int_{0}^{\infty} d t C(t)
$$

In order to estimate $\tau$ more accurately, we also perform an average over 200 independent MC runs, each of which follows the above procedures (4) and (5). In the present study, we measure two macroscopic quantities for $A$ : the total energy $E$ in Eq. (1) and the total magnetization $M \equiv\left|\sum_{j} e^{i \phi_{j}}\right|$.

After we compute the relaxation time $\tau_{E}$ and $\tau_{M}$ for the energy $E$ and the magnetization $M$, we then apply the FSS analysis. The basic idea of the standard FSS is that one can investigate the critical behavior by a systematic observation of simulation results obtained for finite but large-enough various system sizes [12,21-23]. Although the conventional FSS method is based on the scaling behavior of the free energy, one can extend the FSS for the scaling of the relaxation time $\tau$ :

$$
\tau=N^{\bar{z}} \mathcal{F}\left[\left(T-T_{c}\right) N^{1 / \bar{v}}\right],
$$

where $T$ denotes the temperature and $T_{c}$ is the critical temperature. The argument $\left(T-T_{c}\right) N^{1 / \bar{v}}$ of the scaling function $\mathcal{F}$ reflects the ratio between the system size $N$ and the correlation volume $\xi_{V} \sim\left|T-T_{c}\right|^{-\bar{v}}$. Exactly at the critical point, $(T-$ $\left.T_{c}\right) N^{1 / \bar{v}}$ vanishes, and we get $\tau \sim N^{\bar{z}}$ as explained in Sec. I. We use the FSS form of $\tau$ as follows: All curves for different system sizes can be made collapse if we plot $\tau N^{-\bar{z}}$ versus $\left(T-T_{c}\right) N^{1 / \bar{v}}$ when the correct values of $T_{c}, \bar{v}$, and $\bar{z}$ are chosen [17,21,22]. We use $T_{c}=1 / 2$ and $\bar{v}=2$ in Ref. [7] and thus the only remaining parameter to adjust to achieve the scaling collapse is $\bar{z}$.

If the trial angle range $\Delta \phi$ is very small, then the trial angle variable is not much different from the previous value, and thus most of the MC trials are accepted. Accordingly, the smaller $\Delta \phi$, the larger the acceptance ratio, and the relaxation becomes the slower. We thus believe that the relaxation time scales at different trial angle ranges should not be compared directly. To deduct the effect of time scale difference originated from the difference in the acceptance ratio, we introduce the scaled relaxation time $\tilde{\tau}$ by multiplying the acceptance ratio to the relaxation time $\tau$. The relation between $\tau$ and $\tilde{\tau}$ is not trivial, since the acceptance ratio strongly depends on the temperature. In Fig. 1 we display the scaling collapses of both $\tau_{E}\left[(\mathrm{a})\right.$ and (b)] and $\tilde{\tau}_{E}[(\mathrm{c})$ and (d)] at two different values of the trial angle range $\Delta \phi=\pi[(\mathrm{a})$ and (c) $]$ and $\Delta \phi=\pi / 6[$ (b) and $(d)]$. (Although not shown here, we confirm that the use of the magnetization $M$ yields the scaling collapse of similar quality as well.) We observe that the value of the dynamic critical exponent approaches the expected value of $1 / 2$ (i) as $\Delta \phi$ is decreased and (ii) if the MC time is scaled by using the measured acceptance ratio. However, the use of $\tilde{\tau}_{E}$ instead of $\tau_{E}$ does not fully remove the gap between the observed and the expected values of $\bar{z}$, but the effect of trial angle range still remains. As $\Delta \phi$ approaches null value, making the relaxation extremely slow, the dynamic critical exponents measured from both $\tilde{\tau}_{E}$ and $\tau_{E}$ appear to approach $\bar{z}=1 / 2$ as shown in Fig. 2.

\section{B. Short-time relaxation}

There is another way, the so-called the short-time relaxation method $[9,13,14,20]$, to measure the dynamic critical exponent by using relaxation behavior from nonequilibrium to equilibrium. Within this framework, MC simulation at a given temperature starts from the completely ordered phase in which $\phi_{i}=0$ for all $i$ 's, and the system approaches equilibrium state as time proceeds. The key quantity $Q$ to investigate dynamic critical behavior is defined as $[9,13,14,20]$

$$
Q(t, N, T) \equiv\left\langle\operatorname{sgn}\left[\sum_{i=1}^{N} \cos \phi_{i}(t)\right]\right\rangle,
$$

where $\langle\cdots\rangle$ is the average over independent $\mathrm{MC}$ runs starting from the identical initial condition $\left[\phi_{i}(t=0)=0\right.$ for $\left.\forall i\right]$, and $\operatorname{sgn}(x)= \pm 1$ for $x \gtrless 0$. Note that although $\operatorname{sgn}\left[\sum \cos \phi_{i}\right]$ is either +1 or $-1, Q(t)$ can have a continuous value in $[-1,1]$ after the sample average $\langle\cdots\rangle$. The value of $Q$ starts from $Q(t=0)=1$ and eventually approaches $Q(t \rightarrow \infty)=$ 0 , since $\operatorname{sgn}\left[\sum \cos \phi_{i}\right]$ can be either +1 or -1 without any specific preference after a sufficiently long time. The scaling form for $Q$ is written as [20]

$$
Q(t, N, T)=\mathcal{Q}\left[t N^{-\bar{z}},\left(T-T_{c}\right) N^{1 / \bar{v}}\right],
$$


(a) $\Delta \phi=\pi$

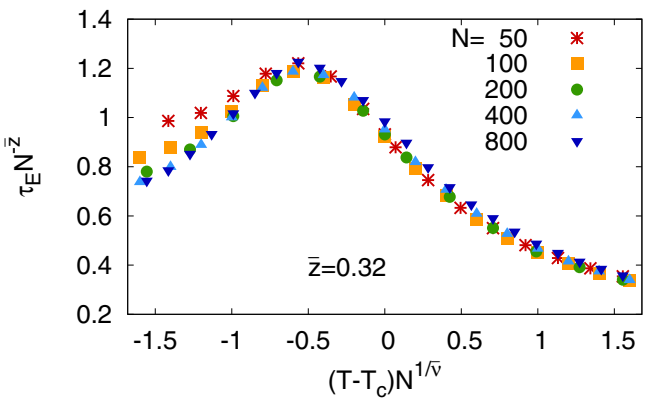

(c) $\Delta \phi=\pi$

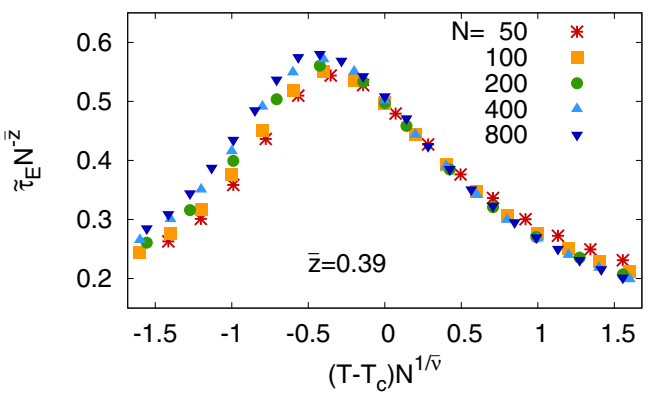

(b) $\Delta \phi=\pi / 6$

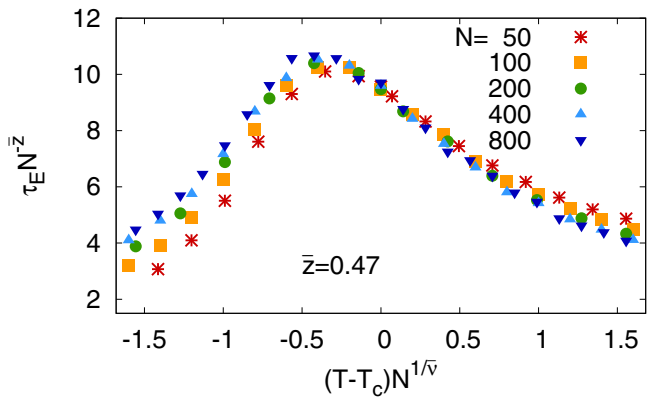

(d) $\Delta \phi=\pi / 6$

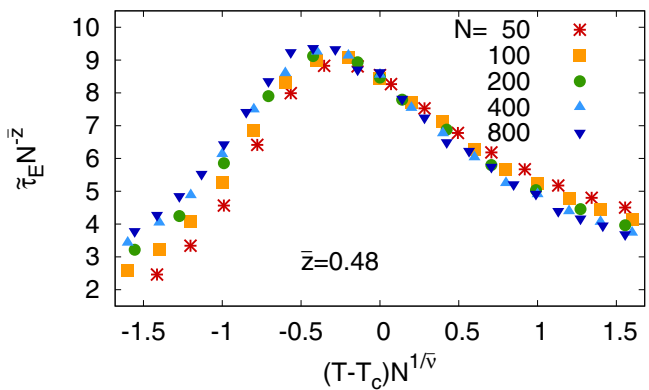

FIG. 1. Finite-size scaling collapse of the relaxation time of the energy for trial angle ranges $\Delta \phi=\pi[(\mathrm{a})$ and (c) $]$ and $\Delta \phi=\pi / 6[(\mathrm{~b})$ and (d)] from the MC simulations. For (a) and (b), we use the MC time in simulation to measure $\tau_{E}$, and for (c) and (d), we use the scaled MC time with respect to the acceptance ratio (see text) to measure the corresponding value of $\tilde{\tau}_{E}$. We observe that the dynamic critical exponent $\bar{z}$ increases as the smaller value of $\Delta \phi$ is used [compare (a) and (b) and then (c) and (d), respectively]. Furthermore, we find that if we scale the MC time in terms of the observed acceptance ratio, then we get $\bar{z}$ closer to the expected value of $1 / 2$ [compare (a) and (c) and then (b) and (d), respectively]. For (a)-(d), we have tried to make curves collapse better near $T=T_{c}$, and $T_{c}=1 / 2$ and $\bar{v}=2$ have been used.

where the scaling variables $t N^{-\bar{z}}$ and $\left(T-T_{c}\right) N^{1 / \bar{v}}$ are related with the finite-time $(\tau / t)$ and the finite-size $\left(\xi_{V} / N\right)$ effects, respectively [20]. Since we are interested in the dynamic critical behavior, we fix the temperature at $T=T_{c}$. The $Q$ becomes the function of ratio of two time scales, $Q\left(t, N, T_{c}\right)=Q\left(t N^{-\bar{z}}\right)$, which yields a simple one-variable scaling form $Q\left(t, N, T_{c}\right)=\mathcal{Q}\left(t N^{-\bar{z}}, 0\right)$. Accordingly, if we

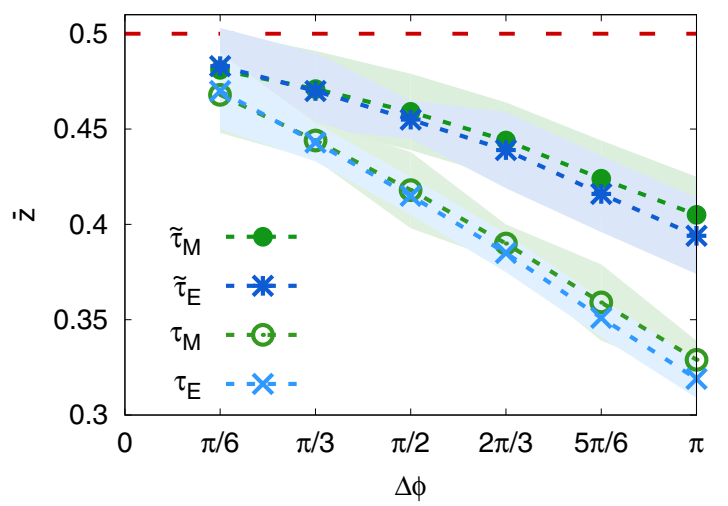

FIG. 2. Dynamic critical exponent $\bar{z}$ versus the trial angle range $\Delta \phi$ for the MC simulations. The relaxation time $\tau$ and the scaled relaxation time $\tilde{\tau}$ for the energy $E$ and the magnetization $M$ are estimated from the FSS method. It is clearly seen that $\bar{z}$ appears to approach $1 / 2$ (the red dashed horizontal line) as $\Delta \phi \rightarrow 0$. The shaded area represents the error bar sizes. plot $Q$ versus $t N^{-\bar{z}}$, then all curves must collapse to a single curve if the proper value of $\bar{z}$ is chosen.

We display our scaling results of the short-time relaxation method in Fig. 3 for $\Delta \phi=\pi$ and $\pi / 6$. Similarly to Fig. 1 for equilibrium investigation, we again find that the smaller value of $\Delta \phi$ gives us the dynamic critical exponent closer to the expected one $(\bar{z}=1 / 2)$. Differently from Fig. 1 where we also present the result for the scaled relaxation time, we do not scale the MC time for $Q(t)$ because the acceptance ratio is not a constant but changes in time as the MC simulation proceeds toward equilibrium. The results of short-time relaxation are consistent with equilibrium results in Sec. III A: The smaller the trial angle range $\Delta \phi$, the closer the dynamic critical exponent $\bar{z}$ to $1 / 2$.

\section{RESULTS: RESISTIVELY-SHUNTED-JUNCTION DYNAMICS}

We present our simulation results from the RSJ dynamics explained in Sec. I. It should be emphasized that both the MC and the RSJ simulations are equivalent if we are interested only in equilibrium property. Both dynamics in equilibrium generate the Boltzmann distribution of the canonical ensemble, i.e., the probability $P\left(\left\{\phi_{i}\right\}\right)$ of the microstate represented by $\left\{\phi_{i}\right\}$ is given by $P \sim e^{-H\left(\left\{\phi_{i}\right\}\right) / T}$ with the Hamiltonian in Eq. (1) and the temperature $T$ in units of $J / k_{B}$ [10]. Although the equilibrium properties cannot be different in principle, dynamic critical behaviors are not guaranteed to be identical. For example, the MC dynamics lacks any realistic time scale 
(a) $\Delta \phi=\pi$

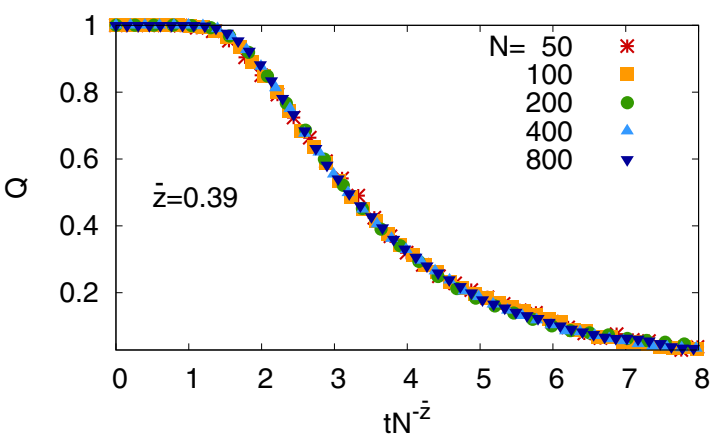

(b) $\Delta \phi=\pi / 6$

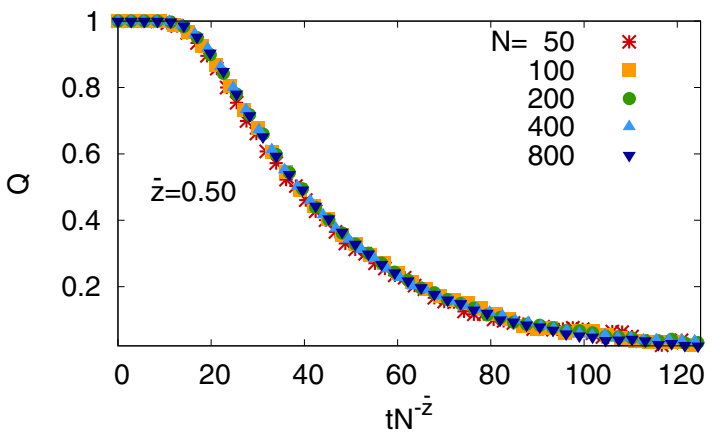

FIG. 3. Short-time relaxation of $Q$ for trial angle ranges (a) $\Delta \phi=\pi$ and (b) $\Delta \phi=\pi / 6$ from the MC simulations. For a sufficiently small value of the trial angle range $[\Delta \phi=\pi / 6$ in (b)], we obtain $\bar{z} \approx 1 / 2$.

to be compared with the real dynamics of the system, and to make it worse, one MC time can sharply depend on the details of the update algorithm in MC simulation. In this regard, we claim that the RSJ dynamics applied for the $X Y$ model is more real than the MC dynamics. The present section is devoted to comparisons of the results in Sec. III for the Metropolis MC simulations with the results from RSJ dynamic simulations.

We numerically integrate the RSJ equations of motion (3) by using the second-order algorithm [10] with the integration time step $\Delta t=0.01$. We disregard the first half of the simulation time until $t=3 \times 10^{4}$ is reached for equilibration, and the data from the other half until $t=6 \times 10^{4}$ are used to compute the autocorrelation function (4) of the energy for the system size up to 40 . For the system sizes $N=60$ and 80 , total simulation time is $t=8 \times 10^{4}$ and the first half of simulation time is used for equilibration and the data from the remaining half are used. To get a better statistics, we also perform an average over 200 independent simulations starting from different initial conditions and different realizations of thermal noise currents. Once the autocorrelation function (4) is measured, we use Eq. (5) to compute $\tau_{E}$. Although we use the fast Fourier transformation algorithm [10] for the lattice Green function in Eq. (3) to make the numerical integration faster, the RSJ dynamics is much slower than the MC simulation. We thus present RSJ simulation results of $\tau_{E}$ only at $T=T_{c}$ in Fig. 4 for system sizes $N=10,15,20,30,40,60$, and 80 . Although the system sizes are much smaller than in Fig. 1 for the MC dynamics, the curve fit to the data points

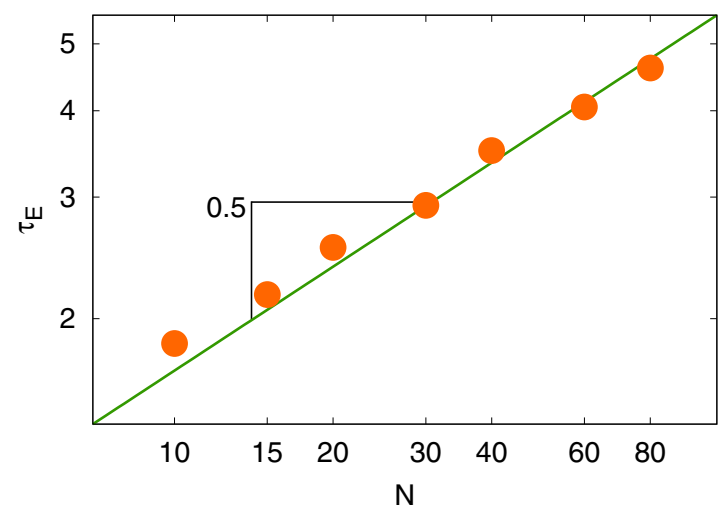

FIG. 4. The equilibrium relaxation time $\tau_{E}$ computed from the autocorrelation function of the energy for the RSJ dynamics for the system sizes $N=10,15,20,30,40,60$, and 80 . We fix the temperature to the critical temperature $T_{c}=0.5$. We use the log scale for both axes in the figure. The slope of the solid line is 0.5 , which is in agreement with the expected value of $\bar{z}=0.5$.

in Fig. 4 yields $\bar{z}=0.50(2)$, which is in agreement with the expected value $1 / 2[13,14]$.

We next apply the short-time relaxation method in Sec. III B and compute $Q(t)$ starting from ordered phase, $\phi_{i}(t=0)=0$ for all $i$ 's. In Fig. 5, we display the scaling of $Q(t)$ for $N=50,75,100$, and 150 , obtained from averages over $2 \times 10^{4}$ independent runs. The best quality of the collapse of curves from different sizes is obtained at $\bar{z}=0.46$, somewhat smaller but consistent with the expected value $1 / 2$.

\section{SUMMARY AND DISCUSSION}

We have numerically studied the dynamic critical behavior of the one-dimensional $X Y$ model with a long-range interaction through the use of the Metropolis Monte Carlo simulations as well as the RSJ dynamic simulations. We have investigated both the dynamic fluctuation in equilibrium and the short-time relaxation from a nonequilibrium initial state toward equilibrium. The equilibrium relaxation time of the autocorrelation function has been measured and the

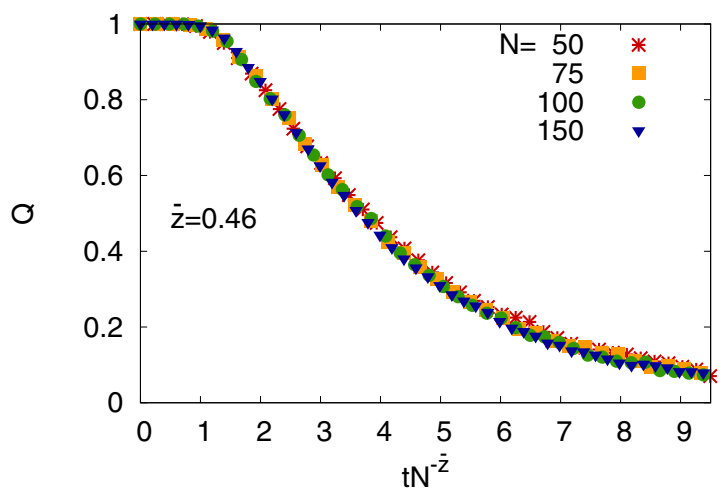

FIG. 5. Scaling collapse of $Q(t)$ at $T=T_{c}=1 / 2$ for system sizes $N=50,75,100$, and 150 from the RSJ dynamics. The dynamic critical exponent $\bar{z} \approx 0.46$ is obtained. 
TABLE I. The dynamic critical exponent of the one-dimensional $X Y$ model with a long-range interaction for the Metropolis MC dynamics (Sec. III) and the more realistic RSJ dynamics (Sec. IV). We have used the finite-size scaling of the relaxation time $\tau_{E}$ for the equilibrium autocorrelation function, and the scaled $\tilde{\tau}_{E}$ by the acceptance ratio has also been used. The finite-time scaling of the quantity $Q(t)$ for the nonequilibrium relaxation toward equilibrium has also been performed.

\begin{tabular}{cccccccc}
\hline \hline & \multicolumn{6}{c}{$\operatorname{MC}(\Delta \phi)$} & \\
\cline { 2 - 6 } & $\pi$ & $5 \pi / 6$ & $2 \pi / 3$ & $\pi / 2$ & $\pi / 3$ & $\pi / 6$ & RSJ \\
\hline$\tau_{E}$ & $0.31(1)$ & $0.35(1)$ & $0.38(1)$ & $0.41(1)$ & $0.44(1)$ & $0.47(2)$ & $0.50(2)$ \\
$\tilde{\tau}_{E}$ & $0.39(2)$ & $0.41(2)$ & $0.43(2)$ & $0.45(1)$ & $0.47(2)$ & $0.49(2)$ & - \\
$Q$ & $0.38(1)$ & $0.42(2)$ & $0.45(1)$ & $0.47(1)$ & $0.49(2)$ & $0.50(1)$ & $0.46(3)$ \\
\hline \hline
\end{tabular}

FSS method has been applied. We have also applied the finite-time scaling [20] method to the key quantity $Q(t)$ for the nonequilibrium short-time relaxation.

We report that the dynamic critical exponent $\bar{z}$ of the model strongly depends on the trial angle range $\Delta \phi$ in the Metropolis Monte Carlo dynamics: The smaller $\Delta \phi$, the larger $\bar{z}$ is obtained. As $\Delta \phi$ approaches null, it appears that $\bar{z}$ approaches $1 / 2$, the expected value for the mean-field universality class. We have also scaled the MC time by using the measured acceptance ratio in MC simulation. Although such scaled MC time yields $\bar{z}$ closer to $1 / 2$, it again exhibits the systematic dependence on $\Delta \phi$. Similar computations have been made in the scheme of the RSJ dynamics which is supposed to be more realistic dynamics than the MC dynamics. We emphasize that both the Metropolis MC dynamics and the RSJ dynamics are equivalent for any of equilibrium quantities since both dynamics yield the identical Boltzmann distribution in equilibrium. Our RSJ simulations have led us to conclude that the equilibrium relaxation time and the short-time relaxation unanimously result in $\bar{z} \approx 0.5$, which is consistent with the theoretical expectation for the long-range $X Y$ model and also with the MC results for the limit of $\Delta \phi \rightarrow 0$. We summarize all the results in the present work in Table I.

We remark that we need to be cautious in the investigation of the dynamic critical behavior via the MC dynamic simulations. More reliable results can be obtained as the temporal evolution is made slow by using a sufficiently small trial angle range of the $X Y$ model. Other dynamics of the $X Y$ model, called the TDGL dynamics $[10,13,14]$, can also be used to study dynamic critical behavior, for which the mathematical representation is identical to the Kuramoto model with the thermal noise. Our result $\bar{z} \approx 0.5$ in the present work is also in agreement with the Kuramoto model with thermal noise [24].

The ordered phase and the disordered phase of the $X Y$ model on the lattice structure can be distinguished from each other by using a suitable nonlocal order parameter [7]. The investigation of dynamic critical behavior with the nonlocal order parameter is a challenging problem which we plan to study in the near future.

\section{ACKNOWLEDGMENTS}

S.-G.Y. and B.J.K. were supported by the National Research Foundation of Korea (NRF) grant funded by the government of Korea (MSIT) (Grant No. 2017R1A2B2005957).
[1] N. D. Mermin and H. Wagner, Phys. Rev. Lett. 17, 1133 (1966).

[2] N. D. Mermin, J. Math. Phys. 8, 1061 (1967).

[3] P. Bruno, Phys. Rev. Lett. 87, 137203 (2001).

[4] J.-S. Yang, K.-I. Goh, I. Mook Kim, and W. Kwak, New J. Phys. 11, 063048 (2009).

[5] B. J. Kim, H. Hong, P. Holme, G. S. Jeon, P. Minnhagen, and M. Y. Choi, Phys. Rev. E 64, 056135 (2001).

[6] J.-S. Yang, W. Kwak, K.-I. Goh, and I.M. Kim, Europhys. Lett. 84, 36004 (2008).

[7] H. Hong and B. J. Kim, Phys. Rev. E 91, 052120 (2015).

[8] D.-R. Ji, J.-B. Zhang, and H.-P. Ying, Commun. Theor. Phys. 20, 477 (1993).

[9] K. Medvedyeva, P. Holme, P. Minnhagen, and B. J. Kim, Phys. Rev. E 67, 036118 (2003).

[10] B. J. Kim, P. Minnhagen, and P. Olsson, Phys. Rev. B 59, 11506 (1999).

[11] R. P. Feynman, R. B. Leighton, and M. Sands, Feynman Lectures on Physics, Vol. III (Addison-Wesley, Reading, MA, 1965).

[12] P. L. Krapivsky, S. Redner, and E. Ben-Naim, A Kinetic View of Statistical Physics (Cambridge University Press, New York, 2010).
[13] L. M. Jensen, B. J. Kim, and P. Minnhagen, Physica B (Amsterdam) 284-288, 455 (2000).

[14] L. M. Jensen, B. J. Kim, and P. Minnhagen, Phys. Rev. B 61, 15412 (2000).

[15] C. J. Lobb, Physica B\&C (Amsterdam) 126, 319 (1984).

[16] K. K. Mon and S. Teitel, Phys. Rev. Lett. 62, 673 (1989).

[17] D. P. Landau and K. Binder, A Guide to Monte Carlo Methods in Statistical Physics (Cambridge University Press, Cambridge, 2000).

[18] M. E. J. Newman and G. T. Barkema, Monte Carlo Methods in Statistical Physics (Oxford University Press, Oxford, 1999).

[19] D. Privman, Finite Size Scaling and Numerical Simulation of Statistical Systems (World Scientific, Singapore, 1990).

[20] M. J. Lee, S. D. Yi, and B. J. Kim, Phys. Rev. Lett 112, 074102 (2014).

[21] N. Goldenfeld, Lectures on Phase Transitions and the Renormalization Group (Addison-Wesley, New York, 1992).

[22] K. Christensen and N. R. Moloney, Complexity and Criticality, Vol. 1 (Imperial College Press, London, 2005).

[23] H. Hong, M. Ha, and H. Park, Phys. Rev. Lett. 98, 258701 (2007).

[24] C. Choi, M. Ha, and B. Kahng, Phys. Rev. E 88, 032126 (2013). 